\title{
NEUES, WISSENSWERTES
}

\section{Eliminierung Antibiotika-resistenter Bakterien mit Low-Level-Laser-Therapie am Beispiel von MRSA im Nasen- und Rachenbereich}

\section{Hintergrund}

Ein Schlüsselerlebnis, die missglückte Eliminierung von MRSA mit Antibiotika bei einem Patienten, gab den Anlass für die Durchführung dieser klinischen Studie mit der Low-Level-Laser-Therapie (Softlaser-Therapie - ,LLLT“).

Die Notwendigkeit für den Versuch der Eliminierung von MRSA sind die weiterhin zunehmende Resistenz von Antibiotika, die steigenden Kosten der Behandlung der Erkrankten und die Zunahme von Todesfällen, was nicht nur nationale, sondern auch weltweite Probleme bereitet. Da eine standardisierte Sanierung mit Antibiotika oft nicht erfolgreich ist, stellt sich die Frage nach Ergänzungen oder Alternativen zu weiteren Sanierungsversuchen, wie die Softlaser-Therapie. Beispiele erfolgreicher Softlaser-Therapien bei MRSAInfektionen, ebenso wie Beispiele, die die Sicherheit dieser Behandlungen dokumentieren, liegen in der wissenschaftlichen Literatur vor.

Angeregt durch die in meiner Praxis schon öfter erfolgreich durchgeführte LLLT kam mir der Gedanke, mit der Therapieoption „Antibakteriell“", Frequenz 425 $\mathrm{Hz}$ (Physiolaser olympic mit Umterzungen-Applikator, Fa. Reimers \& Jansen $\mathrm{GmbH}$ ) einen Therapieversuch zu starten, nachdem mehrfache Antibiotikabehandlungen nach Antibiogramm gescheitert waren. Dieser Therapieversuch war ein sehr ermutigender Erfolg, weswegen ich eine kleine Studie zur Eliminierung generell von MRSA wagte.

\section{Methoden}

Die 14 rekrutierten Probanden mit erfolgloser MRSA-Vorbehandlung wurden über ihre Anamnese erfasst, ausführlich über die Einzelheiten der Studie in- formiert und nach deren Einwilligung im one way crossover design mit sublingualer Einschwingung der Frequenz $425 \mathrm{~Hz}$ (4 Dioden WL 625 nm/2 Dioden $405 \mathrm{~nm}$ ) über die Unterzungenvenen therapiert, das heißt, die Rekrutierten bilden sowohl ihre eigene Kontroll- als auch die Verum-Gruppe, womit weit weniger Patienten als bei einer zweiarmigen Studie benötigt werden. Bei fehlender Sanierung wurden insgesamt bis zu drei LLL-Behandlungen angeschlossen sowie ggf. ein erneuter Sanierungsversuch mit lokalem Antibiotikum und antiseptischer Behandlung.

\section{Ergebnisse}

Die gefundenen Placebo- und Verum-Ergebnisse wurden mit dem McNemar-Test bewertet. Die Auswertung der Placebo- und der kumulierten Verum-Therapien insgesamt ergaben getrennt für den Nasen- und den Rachenbereich eine Signifikanz p mit $p=0,002$ (Nase: von 10 Pat., 9 MRSA frei $=90 \%)$ bzw. $p=0,016$ (Rachen: von 7 Pat., 6 MRSA frei $=86 \%$ ). Es gab Hinweise, dass bei Einsatz von Curcumin die Elimination sicherer und schneller erreicht werden konnte als bei Probanden, denen wegen Kontraindikation diese Substanz nicht gegeben werden konnte.

Bezüglich der Lebensqualität fand sich in keinem Fall eine Verschlechterung, aber bei einzelnen Probanden und Items wurden Verbesserungen beobachtet. Insgesamt blieb die Lebensqualität weitgehend unverändert.

So weisen die vorliegenden Ergebnisse darauf hin, dass die LLLT bei bisher erfolglosen MRSA-Sanierungsversuchen eine zusätzliche Möglichkeit der Erregerelimination bietet und gut verträglich ist. Wenn möglich, sollte dabei Curcumin als Sensitizer eingesetzt werden. In einer größeren Studie sollte geprüft werden, ob sich das Ergebnis bestätigen und quantifizieren lässt sowie auch über 12 Monate nachhaltigen Erfolg zeigt.

Literatur beim Verfasser

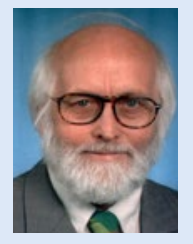

Wolfgang Gregorovius

Poststraße 1

34537 Bad Wildungen 